\title{
Effect of the LncRNA GAS5-MiR-23a-ATG3 Axis in Regulating Autophagy in Patients with Breast Cancer
}

\author{
Juan Gua,b Yueping Wang ${ }^{a, c, d} \quad$ Xuedong Wang ${ }^{a, b, d} \quad$ Daoping Zhou ${ }^{d}$ \\ Xinguo Wang ${ }^{a, b}$ Ming Zhou ${ }^{e}$ Zhimin $\mathrm{He}^{f}$
}

aDepartment of Medical Laboratory Science, The Fifth People's Hospital of Wuxi, The Medical School of Jiangnan University Wuxi, Jiangsu, 'Department of Pathology, The Fifth People's Hospital of Wuxi, Nanjing Medical University, Wuxi, Jiangsu, China, 'Department of Biology, College of Arts \& Science, Massachusetts University, Boston, MA USA, dDepartment of Oncology, The Second People's Hospital of Anhui Province, Anhui Medical University, Hefei, Anhui, 'Cancer Research Institute, Central South University, Changsha, Hunan, ${ }^{\mathrm{f} C a n c e r ~ H o s p i t a l}$ and Cancer Research Institute, Guangzhou Medical University, Guangzhou, Guangdong, China 